\title{
Novel Wafer Bevel Treatment for Water Immersion Lithography
}

\author{
Mamoru Terai ${ }^{1}$, Takeo Ishibashi ${ }^{2}$, Takuya Hagiwara ${ }^{2}$, Tetsuro Hanawa ${ }^{2}$, \\ Teruhiko Kumada', Yoko Takebe ${ }^{3}$, Osamu Yokokoji ${ }^{3}$, Tomoharu Fujiwara, \\ Jianhai Jiang ${ }^{4}$, Takafumi Niwa ${ }^{5}$, Shinya Wakamizu ${ }^{5}$ and Hideharu Kyouda ${ }^{5}$ \\ ${ }^{1}$ Mitsubishi Electric Corp., 8-1-1 Tsukaguchihonmachi, Amagasaki, Hyogo 661-8661, Japan; \\ ${ }^{2}$ Renesas Technology Corp., 4-1 Mizuhara, Itami, Hyogo 664-0005, Japan; \\ ${ }^{3}$ Asahi Glass Co., Ltd., 1150 Hazawa, Kanazawa-ku, Yokohama, Kanagawa 221-8755, Japan; \\ ${ }^{4}$ Nikon Corp., 201-9 Miizugahara, Kumagaya, Saitama 360-8559, Japan. \\ ${ }^{5}$ Tokyo Electron Kyushu Ltd., 1-1, Fukuhara, Koshi City, Kumamoto, 869-1116, Japan
}

\begin{abstract}
In this study, we focus on the controllability of a wafer bevel from adhesion and hydrophobicity viewpoints in order to solve the problems of film peeling and microdroplet formation around wafer bevels, which can result in pattern defects and degrading of the overlay and focus accuracy because of local temperature variation around several sensors due to the vaporization heat of the leaked water. Hexamethyldisilazane (HMDS) treatment is a common solution to these problems. We examine a novel wafer bevel treatment utilizing silane coupling agents ( $\mathrm{SCa}$ ) for obtaining high hydrophobicity and adhesion. These groups react with inorganic substrates and films on the surface that are subjected to a novel chemical treatment (NCT). The coating performance of chemicals on the surface by the NCT and the aging stability of the formulated solution of the SCas are examined for optimizing the composition of the NCT solution. We revealed that the NCT agent, which is comprised of long-chain alkyl type SCa and additive (i.e. acid and salt), has good performance. Furthermore, we verified the superiority of using NCT in the water meniscus stability with high scanning speed (i.e. $500 \mathrm{~mm} / \mathrm{sec}$ ) and water leakage in wafers with a topcoat, ArF resist, and bottom antireflective coating (BARC) using a quasi-immersion exposure stage.
\end{abstract}

Keywords: immersion lithography, wafer bevel, film peeling, hydrophobicity

\section{Introduction}

The mass production of a half pitch $45 \mathrm{~nm}$ node device has already been established by using argon fluoride immersion lithography (IML). This process utilizes topcoats, which prevents elution from resists and obtains surfaces with a large receding contact angle (RCA) in order to maintain PR performance and water mark defect [1]. Recently, most photoresist (PR) vendors have begun to develop non-topcoat resists, and IML tool vendors are making aggressive efforts to develop high-speed scanning exposure tools to obtain the cost advantage of the IML process, which has several issues. One of them is the particle problem of film peeling around the wafer bevel. Such peeling is caused by an inadequate adherence between the surface around the wafer bevel and the film coated on the surface. Hexamethyldisilazane (HMDS) treatment is usually employed for improving this poor adherence. However, the peeling of the topcoat film, shown in
Fig. 1(a), has been reported even for this conventional method [2-3]. This result indicates that the adherence, which is obtained by HMDS treatment, is not always sufficient. Furthermore, it should be noted that it is difficult to form a stable water meniscus around the hydrophilic surface at the wafer bevel. Therefore, there is another problem with the formation of water microdroplets around the wafer bevel, which has insufficient hydrophobicity (Fig. 1(b)). It has the possibility that leaked water reaches the bottom of the wafer and damages the wafer stage [4]. Furthermore, this phenomenon has possibilities of degrading the overlay and focus accuracy because of local temperature variation around several sensors due to heat of vaporization of the leaked water. 

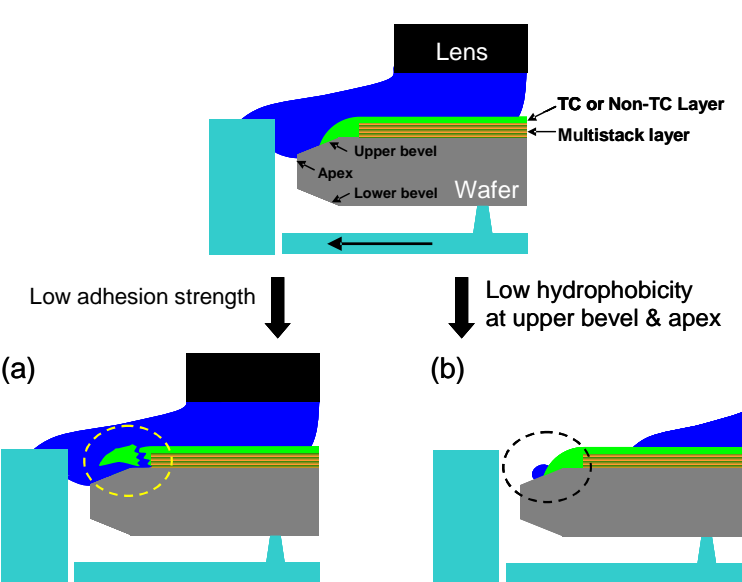

Low hydrophobicity at upper bevel \& apex

(b)

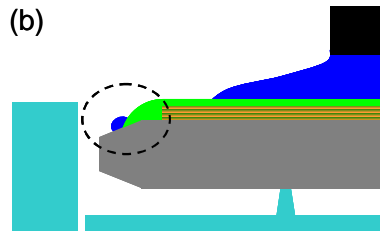

Fig. 1. Drawbacks of immersion process at wafer bevel. (a) Film peeling due to low adhesion between wafer and coating film and (b) water microdroplets due to insufficient hydrophobicity.

From the viewpoint of maintaining a stable water meniscus at the wafer bevel portion, the films should be coated such that the surface of the edge of the upper bevel is also coated, as shown in Fig. 1. From the viewpoint of preventing the particles by any ineluctable contacts of the wafer bevel in transportations in track (i.e., coater and developer) and the exposure tool, as a minimum, the coating film on the upper bevel should be removed. The controlling of the coverage of the coating films that satisfies both demands mentioned above is very difficult. In consequence, the apex and some adjacent areas of the bare wafer bevel, which are not covered by a highly hydrophobic coating film, would be exposed to the meniscus of the immersion water in the exposure tool moving at a high scanning speed. Moreover, in the process of preparing a non-topcoat resist, the total area of the wafer bevel surface will be exposed to the immersion water in the case of a cut edge of the resist, which is probably a requirement for the manufacturing of the device. It is possible that the hydrophobicity of the wafer bevel by the conventional HMDS treatment is insufficient for dealing with advancements such as non-topcoat resists and higher-scanning-speed exposure tools. The method for obtaining a hydrophobic surface around the wafer bevel requires careful consideration. In this article, we wish to report on the progress of the development of a novel chemical treatment (NCT).

\section{Experimental}

\subsection{Material}

We determine the efficient utilization of the chemical structure of a bone, comprising trimethoxysilanol and organic functional groups as shown in Fig. 2(a). Because this bone structure has a wide variety of organic functional groups, which are denoted by " $X$ " in Fig. 2(a), this advantage makes it possible to chemically adjust the hydrophobicity and adhesion properties with comparative ease. Almost none of the organic functional groups used in this study exhibit reactivity; however, for convenience, this chemical structure is hereinafter referred to as a "silane coupling agent (SCa)". All SCa samples (by Asahi Glass Co., Ltd.) tested in this study were prepared using propylene glycol monomethyl ether acetate (PGMEA) as a solvent, and were used within 3 days from the time of preparation.

(a)

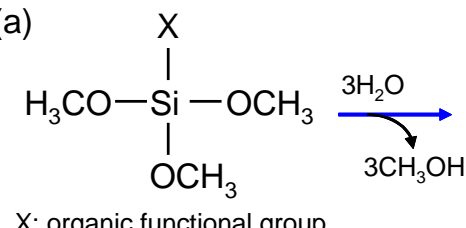<smiles>[X][Si](O)(O)O</smiles>

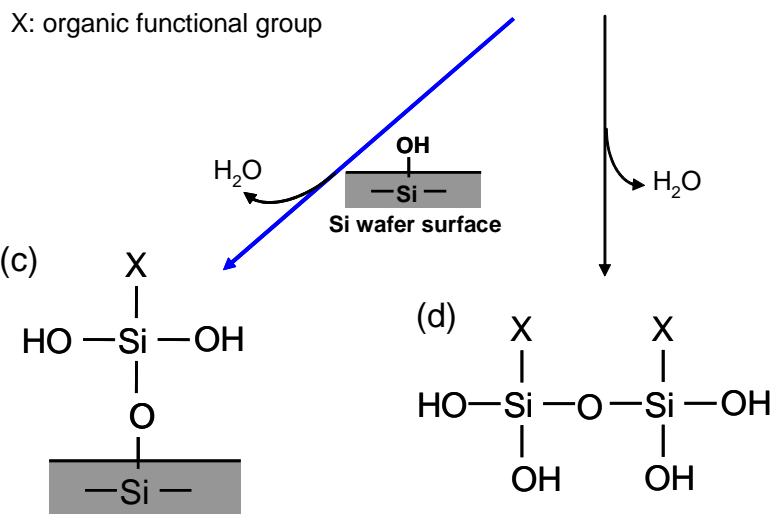

Fig. 2. Reaction of silane coupling agent. (a) silane coupling agent, (b) silanolization by hydrolysis reaction, (c) attachment reaction with Si wafer, and (d) oligomerization.

The well-known chemical reaction scheme [5] of the $\mathrm{SCa}$ is shown in Fig. 2. Methoxysilanol is selected because of its high reactivity among all alkoxy groups. First, methoxysilanol is hydrolyzed to silanols, which can react with an inorganic surface by hydrogen bonding (Fig. 2(b)). These hydrolyzed silanols can undergo two possible reactions-dehydration reactions with the silanols of the inorganic surface (Fig. 2(c)) and the reaction among the silanols of other individual SC agent molecules; the latter reaction is generally referred to as oligomerization (Fig. 2(d)). 


\subsection{Experimental Equipment and Conditions}

In the feasibility study and investigation of the properties of several SCa, a simple standalone manual coating spinner was used. Model CA-X manufactured by Kyowa Interface Science Co., Ltd, was used to measure static and dynamic contact angles (CA). The adhesion strength was measured using a tensile yield stress tester developed in-house, composed of a stud with a $3 \mathrm{~mm}$ diameter and a digital push-pull gage (RX-10, Aikoh Engineering Co., Ltd.) connected to the stud. The measurements were performed as follows. The $3 \mathrm{~mm}$ surface of the stud was attached to a previously coated topcoat surface by adhesion bonding and left to rest for at least $12 \mathrm{~h}$. The stud was pulled upward perpendicularly after immersing the area around the stud in deionized water (DIW) as shown in Fig. 3.

The actual scanning test was performed using a double-sided polished $300 \mathrm{~mm}$ wafer. The SCa sample was installed in the edge rinse (ER) and back rinse (BR) lines of the CLEAN TRACK $\mathrm{ACT}^{\mathrm{TM}} 12$ (ACT12) manufactured by Tokyo Electron Ltd. (TEL). The fluid delivery rates of the ER and BR lines were set to the standard value used by TEL. The NCT was carried out at a distance of $10 \mathrm{~mm}$ from the wafer bevel in order to determine the CA of the wafer bevel at the $5 \mathrm{~mm}$ position. The feeding time of the SCa and the rotation speed were optimized to 1.5 30 s and 500 2000 rpm, respectively. Baking treatment at 150 degree for one minute was performed to enhance the dehydration reaction after the feeding. Water-meniscus-scanning tests were performed using a simplified scan stage module manufactured in the laboratory of Nikon Corp.

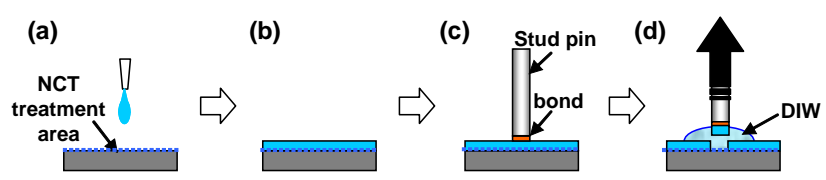

Fig. 3. Method of adhesion strength: (a) topcoat spin coating, (b) baking, (c) attachment of stud pin, and (d) measurement of adhesion strength.

\section{Results and discussion}

\subsection{Selection of SCa for NCT agent}

Our focus is from the basic reactivity of the SCa, dominated by the trimethylsilanol group, to the total performance of the SCa with regard to its suitability for use as an NCT agent. The performance parameters considered in this study are the hydrophobicity of the treated surface, adhesion strength of the topcoat, and various promising coating materials. In addition, we consider the cost and environmental safety of the primary material for the NCT agent. We revealed that several parameters (i.e. tuning concentration of SCa, water content, and acidity of solution) can control the attachment reaction between SCa and the Si-wafer [6]. We investigate the influence of the chemical structure on the attachment reaction. In particular, the length of the alkyl group and the presence of fluorine were focused on. The tested sample list is shown in Table 1.

Table 1. Investigated NCT samples for determining the influence of chemical structure of organic functional groups of SCa.

\begin{tabular}{|c|c|c|c|c|}
\hline \multirow{2}{*}{$\begin{array}{l}\text { Sample } \\
\text { name }\end{array}$} & \multicolumn{3}{|c|}{$\mathrm{SCa}$} & \multirow{2}{*}{$\begin{array}{c}\text { Acid } \\
\text { [molar rati } \\
\text { to SCa] }\end{array}$} \\
\hline & functional group & chain length & $\begin{array}{c}\text { conc. } \\
\text { [normalized] }\end{array}$ & \\
\hline Sample-F-A & \multirow{2}{*}{ Fluoro-alkyl type } & \multirow{2}{*}{$\begin{array}{l}\text { very short } \\
\text { short }\end{array}$} & 1 & 0.01 \\
\hline Sample-F-B & & & 1 & 0.01 \\
\hline Sample-A-A & \multirow{4}{*}{ Alkyl type } & short & 1 & 0.01 \\
\hline Sample-A-B & & middle & 1 & 0.01 \\
\hline Sample-A-C & & long & 1 & 0.01 \\
\hline Sample-A-D & & longest & 1 & 0.01 \\
\hline
\end{tabular}

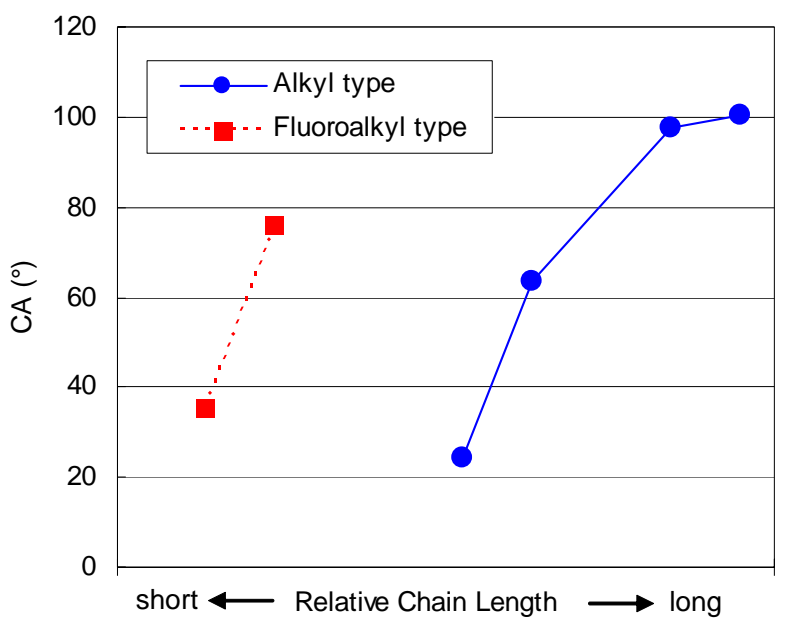

Fig 4. Static CA on the surface treated by NCT agents with various chemical structures, both with and without fluorine. 
Table 2. NCT sample list and result of investigation of the influence of formulation parameters (i.e., SCa concentration and amount of added acetic acid) on static CA and adhesion strength.

\begin{tabular}{ccccc||cc}
\multirow{2}{*}{$\begin{array}{c}\text { Sample } \\
\text { name }\end{array}$} & functional group & $\begin{array}{c}\text { Concentration } \\
\text { [normalized] }\end{array}$ & $\begin{array}{c}\text { amount of acid } \\
\text { [molar ratio to }\end{array}$ & CA $\left({ }^{\circ}\right)$ & $\begin{array}{c}\text { Adhesion } \\
(\mathrm{N} / \mathrm{mm} 2)\end{array}$ \\
\cline { 2 - 5 } & & 1 & 0.01 & 46.8 & 6.8 \\
\hline Sample-A-D & longest-chain alkyl type & 1 & 0.19 & 66.2 & 3.4 \\
Sample-A-E & $\begin{array}{l}\text { longest-chain alkyl type } \\
\text { Sample-A-F }\end{array}$ & $\begin{array}{l}\text { longest-chain alkyl type } \\
\text { Sample-A-G }\end{array}$ & 1 & 0.48 & 74.0 & 1.3 \\
\hline Sample-F-B & longest-chain alkyl type & 2 & 0.10 & 78.7 & 1.2 \\
Short fluoroalkyl type & 1 & 0.01 & 21.6 & 5.7 \\
Sample-F-C & short fluoroalkyl type & 1 & 0.19 & 46.3 & 1.2 \\
Sample-F-D & short fluoroalkyl type & 1 & 0.47 & 54.6 & 0.9 \\
\hline HMDS & short fluoroalkyl type & 2 & 0.09 & 41.7 & 1.0 \\
\hline
\end{tabular}

To standardize the conditions of these tests, the acidity is set to $\mathrm{pH} \sim 4$ by adding acetic acid with a concentration of $0.2 \sim 2.5 \mathrm{wt} \%$ (ca. 2E-3 4E-3 $\mathrm{mol} / \mathrm{l}$ ), which is generally considered to be a stable $\mathrm{pH}$ zone for silanols; furthermore, the water contents are set to approximately $3.5 \mathrm{E}-3 \mathrm{~mol} / \mathrm{l}$ in order to adjust the normality of the silanol group in the solution.

Fig. 4 clearly shows that the static CA increases with an increase in the alkyl group length, and the static CAs of the fluoroalkyl groups are comparatively larger than those of the alkyl groups. This result is very reasonable in terms of the relationship between the surface composition and critical surface tension [7].

3.2. Formulation parameters of hydrophobicity and adhesion strength

Table 2 summarizes the result of the investigation of the influence of the formulation parameters on the static CA and adhesion strength. In this study, we select SCa with the longest-chain alkyl (LCA) group of sample-A-D and the short-chain fluoroalkyl (SFA) group of sample-F-B as the samples. From Table 2, we observe that the static CA increases with the amount of added acid and the concentration of the SCa itself. Here, we comment on the difference between the absolute values of the static CAs obtained for the same sample-A-D in the two experiments shown in Fig. 4 and Table 2. This difference is the result of several differences in the experimental conditions. In a recent investigation of these experimental factors, we have found that a contamination in experimental material is one of the important error factors. We think that it is possible to exploit such contaminated material for the optimization of the NCT agent used in this study.

The adhesion strength is tested using various formulations for SCa composed of the LCA and SFA groups. The results for the LCA and SFA samples are plotted in Fig. 5. The adhesion strength decreases with an increase in the static CA for the cases of both the LCA and SFA samples. A comparison of the adhesion strengths of the LCA and SFA samples at the same static CA reveals that the adhesion strength of the LCA sample is greater than that of not only the SFA sample but also the HMDS sample. Therefore, we can say that the NCT carried out by utilizing the SCA of the LCA group provides better adhesion than that obtained by the conventional HMDS treatment.

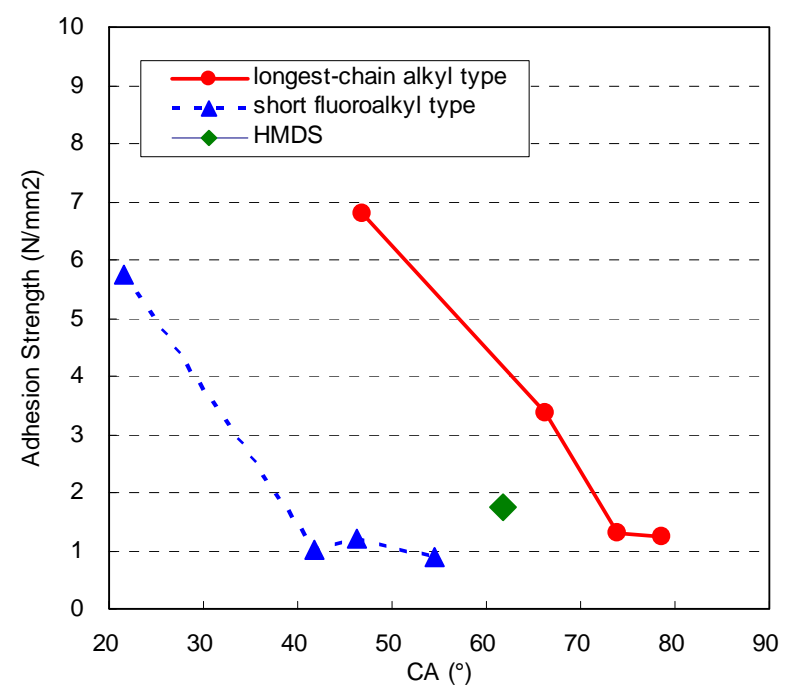

Fig. 5. Dependency of adhesion strength and static CA on the surface treated by NCT agents with various chemical structures both with and without fluorine. 
Next, we tested the influenced of salts $(\mathrm{R}+\mathrm{X}-)$ as an additive of NCT agent to improve attachment reaction to the Si-wafer by created silanoxide $\left(\mathrm{Si}-\mathrm{O}^{-} \mathrm{R}^{+}\right)$. The NCT agent used was longest-chain alkyl type SCa, acetic acid and acidic salt. The concentration of each of the contents is shown in Table 3.

Fig. 6 shows the results of static CA depending on the addition of salt. The SCa and acid concentration of both samples from Table 4 are similar to that of sample-A-D, which has low hydrophobicity (Table 2). Therefore the static CA of sample-A-H is a level equal to that of sample-A-D. However, the static CA of sample-A-I, which has salt added, is over $100^{\circ}$. This result shows that not only the acid but also the salt is effective for the formulation parameters of hydrophobicity.

Table 3. List of tested NCT agent.

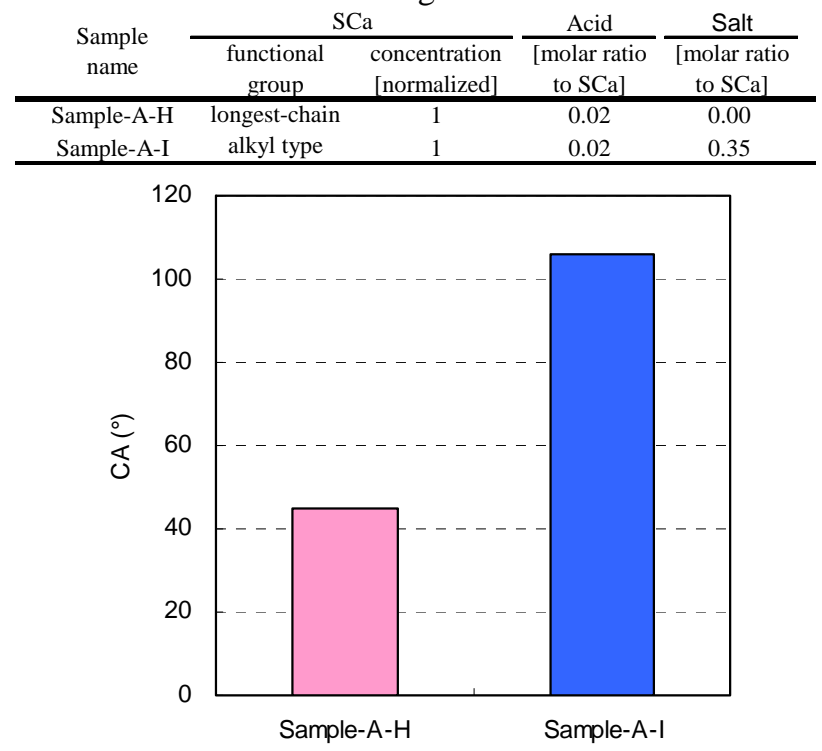

Fig. 6. Static CAs of NCT agent with / without salt.

Fig. 7 shows the relationship between the static CA and the blend ratio of two kinds of SCas. We used short-chain alkyl type SCa of sample-A-A and longest-chain alkyl type SCa of sample-A-D as shown in Table 1 as blended SCa in this evaluation. The concentrations of added acid and salt are the same as sample-A-I in Table 3. In the result, the blend ratio of longest-chain alkyl type SCa decreases with a decrease in the static CA. A comparison of the blend ratio of the longest-chain and short-chain alkyl type SCa reveals that the hydrophobicity is maintained even if the ratio of short-chain alkyl type increases to about 90 mol\%. Therefore, we can get the variety of hydrophobicity of the wafer bevel by blending the SCa with the different hydrophobicity.

Next, we examined the reactivity between the NCT agent and the Si wafer bevel. Fig. 8 shows the relationship between the static CA and the treatment volume of the new NCT agent and the first trial SCa samples. These samples had controlled concentration of the component to match the reciprocal hydrophobicity. This result shows that the new NCT sample has higher reactivity than the first-trial NCT sample.

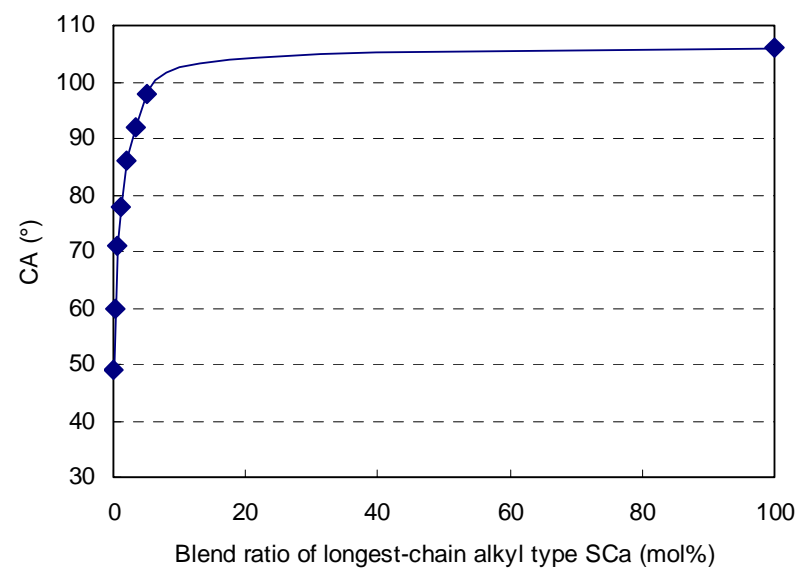

Fig. 7. Relationship between static CA and blend ratio of two types of SCa.

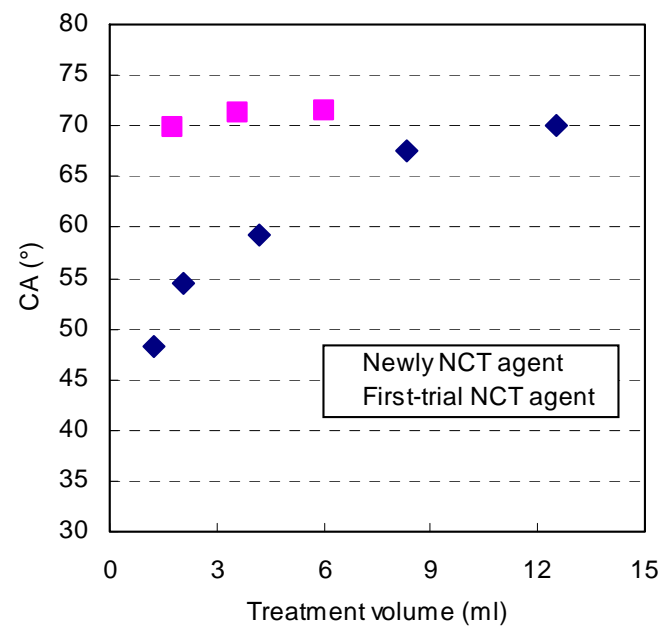

Fig. 8. Relationship between static CA and treatment volume of two types of NCT agents. New NCT agent consists of blended LCA (longest:short $=2: 98$, LCA conc. $=1$ [normalized value], acid $=0.02$ [molar ratio to LCA], salt $=0.35$ [molar ratio to LCA]). First-trial NCT agent consists of prototype SFA samples. 
A crucial performance parameter that should be discussed is the aging stability of NCT agents, which is related to their shelf life. The method by which NCT agents are prepared, i.e. in a manufacturing plant or just before use in a custom-built mixer on a wafer-processing floor, depends on the shelf life. From the results of the number of liquid particles shown in Fig. 9, we can clearly observe that the LCA samples have a longer shelf life than the SFA samples. On observing the LCA-type NCA agent after a duration of 35 days, we find that the solution is cloudy; therefore, we deduce that particles are formed at an accelerating rate after about a month of aging.

On the other hand, we also checked the shelf life of the new NCT agent. We observed that the solution of new NCT agent is absolutely clear after a duration of 10 days.

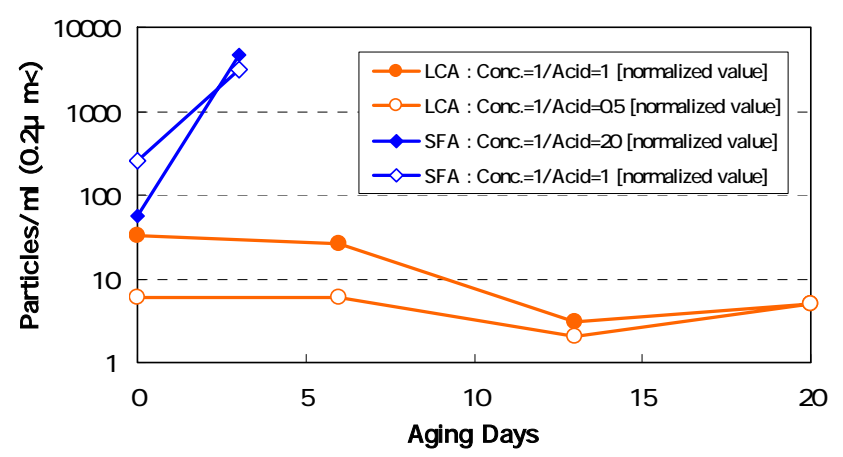

Fig. 9. Trend of number of liquid particles for standard LCA and SFA samples

The next parameter to be evaluated is the coating capability on the NCT-treated surface. Because the NCT-treated surface exhibits a water-shedding property, the surface also sheds the coating liquid. Thus, the coating film cannot cover the entire wafer. Therefore, we checked the coating capability by using NCT agents as shown in Table 2. From Table 4 , we can observe that at the time of coating the surfaces, the possible static CA up to which coating can be carried out for the LCA sample is greater than that for the SFA sample. In other words, the LCA sample can be coated until a static CA of $74.0^{\circ}$ in contrast to a static CA of $21.6^{\circ}$ in the case of the SFA sample. It is important that this quantitative comparison is based on the static CAs of water as a polar solvent.

The coating capability should be discussed on the basis of the total surface energy by considering factors such as dipoles, polarizability, and hydrogen bond elements. We presume that the LCA group is more efficient than the SFA group due to the former group's polarizability and hydrogen bond elements, which are considered in the discussion of the total surface energy. Accordingly, the LCA group has a relatively better coating capability despite having high hydrophobicity.

Table 4. Results of determination of coating capability on the surface treated by the NCT agents listed in Table 2 .

\begin{tabular}{|c|c|c|c|c|c|}
\hline $\begin{array}{c}\text { Treatment } \\
\text { Agent }\end{array}$ & $\mathrm{CA}\left({ }^{\circ}\right)$ & ArF Resist & BARC-A & BARC-B & Topcoat \\
\hline \multirow{4}{*}{$\begin{array}{l}\text { longest-chain } \\
\text { alkyl type }\end{array}$} & 46.8 & OK & OK & OK & OK \\
\hline & 66.2 & OK & $\mathrm{OK}$ & OK & $\mathrm{OK}$ \\
\hline & 74.0 & $\mathrm{OK}$ & $\mathrm{OK}$ & $\mathrm{OK}$ & $\mathrm{OK}$ \\
\hline & 78.7 & OK & OK & NG & OK \\
\hline \multirow{4}{*}{$\begin{array}{l}\text { short fluoroalkyl } \\
\text { type }\end{array}$} & 21.6 & OK & OK & OK & OK \\
\hline & 41.7 & $\mathrm{OK}$ & OK & $\overline{N G}$ & OK \\
\hline & 46.3 & OK & NG & NG & $\mathrm{OK}$ \\
\hline & 54.6 & OK & NG & NG & OK \\
\hline HMDS & 61.7 & OK & OK & $\mathrm{OK}$ & $\mathrm{OK}$ \\
\hline
\end{tabular}

3-3. Scanning Test of $300 \mathrm{~mm}$ Wafer using Scan Stage Module

A wafer with a diameter of $300 \mathrm{~mm}$ is subjected to NCT treatment by using sample-A-I (Table 3 ). The machine ACT12 manufactured by TEL is employed in this experiment. The design of two different film structures around the wafer bevel is shown in Fig. 10. A topcoat that is in direct contact with the $\mathrm{Si}$ wafer bevel subjected to the NCT is suitable for investigating the adhesion strength, as shown in Fig. 10(a). A bare Si wafer bevel without a coating film is suitable for verifying the effectiveness of the NCT. It should be noted that only monomolecular layers are actually formed at the NCT treatment area, although a blue dot line of the NCT area is shown in the figure for ease of understanding.

(a)

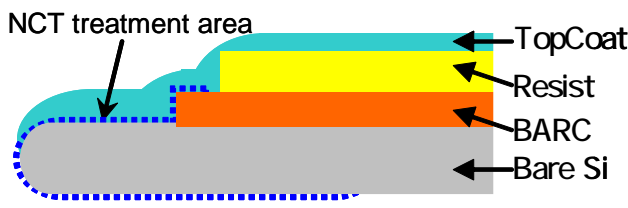

(b)

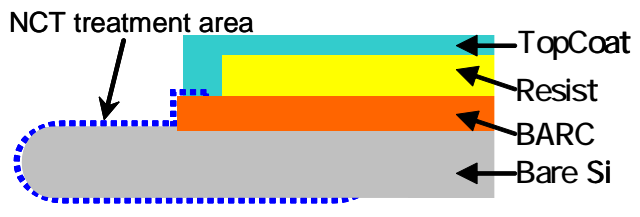

Fig. 10. Structures of wafer bevel used in actual immersion tests. (a) Structure for peeling test of topcoat, (b) Structure for water leakage reaching bottom of wafer 
The water-meniscus-scanning tests are performed using the simplified scan stage module from Nikon Corp. In this scanning test, we evaluate the resistance to topcoat peeling and water leakage. Additionally, we investigated the maximum scanning speed test without emergence of unfavorable droplets using the total main flat surface of the Si wafer with no coating film that formed NCT layer all over the wafer. The results of the scanning test are summarized in Table 5. In the results, the NCT agent has high performance in comparison with the HMDS treatment. A maximum scan speed of 500 $\mathrm{mm} / \mathrm{sec}$ was reached at the NCT treatment wafer. Moreover, no microdroplet was observed. On the contrary, the conventional method has a maximum scan speed of $150 \mathrm{~mm} / \mathrm{sec}$ and some microdroplets were observed.

Table 5. Summary of results of actual immersion scanning test.

\begin{tabular}{ccccccc}
\hline \multirow{2}{*}{$\begin{array}{c}\text { Treatment } \\
\text { material }\end{array}$} & $\begin{array}{c}\text { Static } \\
\text { CA }\end{array}$ & $\begin{array}{c}\text { Receding } \\
\text { CA }\end{array}$ & $\begin{array}{c}\text { Peeling } \\
\text { of } \\
\text { TopCoat }\end{array}$ & $\begin{array}{c}\text { Microdroplet } \\
\text { of } \\
\text { Bottom }\end{array}$ & $\begin{array}{c}\text { Maximun } \\
\text { scan speed } \\
\text { (mm/sec) }\end{array}$ \\
\hline \hline NCT agent & 108.6 & 102.5 & None & None & 500 \\
\hline HMDS & 71.5 & 64.2 & None & Some & 150 \\
\hline
\end{tabular}

\section{Conclusion}

We have investigated the NCT process in order to utilize the water immersion lithography technology, thereby achieving high stability of scanning accuracy and throughput with low cost for mass production; the NCT process utilizes a high scan-speed exposure tool and a non-topcoat resist. This process has been developed to achieve high hydrophobicity and adhesion strength around the wafer bevel in order to make provisions for new requirements in the future.

First, we investigated certain chemical properties of the $\mathrm{SCa}$ selected for this process. On the basis of our past experimental results, we selected $\mathrm{SCa}$ composed of the LCA group and optimized the SCa concentration and amount of acid by taking care of the shelf life of the NCT agent [6]. Moreover, we revealed that the salt addition and blend between SCas, which have respectively different hydrophobicity, are excellent means to control hydrophobicity. In addition, we can say that the LCA group is very cost effective and environmentally safe in comparison with the SFA group.

We obtained a considerable improvement in the film adhesion and hydrophobicity with the NCT, and the actual immersion scanning tests showed consistent results.

In the new NCT agent, which was created by blending LCA and some additives, we made only a visual check of liquid and static CAs in terms of shelf life. We are evaluating it on this point now. Additionally, we are beginning to examine NCT treatment by using a bevel rinse nozzle [8]. It is possible to carry out an NCT treatment simultaneously with the spin coating step of litho-materials (i.e. resist, Si-BARC) instead of bevel rinse thinner (Fig. 11). This advanced process will contribute to productive efficiency in terms of takt time. In conclusion, in the near future, we intend to design a high-performance, low-cost NCT agent that has high reactivity and the shelf life necessary for mass production.

(a)

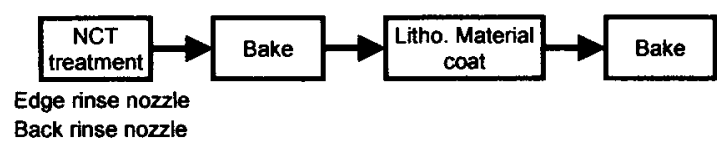

(b)

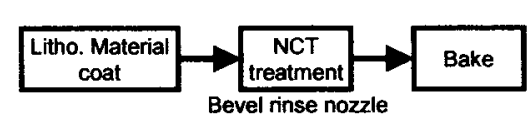

Fig.11 Methods of NCT treatment. (a) Current NCT treatment process, (b) Advanced NCT process.

\section{Acknowledgments}

Firstly, we would like to thank all members of the lithography development group in Renesas Technology Corp. for their support. We also thank the technicians at the Advanced Technology R\&D Center of Mitsubishi Electric Corp. for their assistance with the experiments performed in this study.

\section{References}

1. Yabuuchi, M. et al., ISSCC, 18 (2007) 3.

2. Mulkens, J., Streefkerk, B., Jasper, H., Klerk, J., Jong, F., Levasier, L. and Leenders M., Proc. SPIE, 6520 (2007) 652005.

3. D. Kawamura, T. Takeishi, K. Matsunaga, E. Shiobara, Y. Oonishi and S. Ito, Proc. SPIE, 6153 (2006) 61531Q.

4. S. Owa, H. Nagasaka, Y. Ishii, O. Hirakawa and T. Yamamoto, Proc. SPIE, 5377 (2004) 264.

5. Y. Ohkatsu, ed. Research and Development of Polymer Additive. CMC Publishing Co., Ltd., Tokyo, 1998.

6. I. Takeo, M. Terai, T. Hagiwara, T. Kumada, T. 
Hanawa, Y. Takebe, O. Yokokoji, T. Fujiwara and $\mathrm{H}$. Akiyama, Proc. SPIE, 6923 (2008) 6923-72 to be published.

7. E. F. Hare, E. G. Shafrn, and W. A. Zisman, J.
Phys. Chem., 58 (1954) 236.

8. K. Ichino, K. Tanouchi, T. Iseki, N. Ogata, T. Yamamoto, K.Yoshihara and A. Fujimoto, Proc. SPIE, 6923 (2008) 6923-67 to be published. 